\title{
Selbstbildnis mit fiedelndem Tod
}

Die erste Ausgabe der „Onkologischen Welt" in 2018 begrüßt den Leser auf ihrer Titelseite mit einer zugegebenermaßen nicht mehr ganz zeitgemäßen, aber sehr dramatischen Darstellung zu der ewigen Auseinandersetzung zwischen Krankheit und Tod, der wir uns in der Onkologie in besonderer Weise stellen müssen. Das Bild ist ein Ausschnitt aus dem „Selbstbildnis mit dem fiedelnden Tod" von Arnold Böcklin, einem der großen Symbolisten in der Malerei des 19. Jahrhunderts.

Auch in Böcklins Leben lagen Stationen der künstlerischen Entwicklung und des familiären Leids eng beieinander. Acht seiner 14 Kinder starben früh, er selbst starb 1901, nachdem er mehrere Schlaganfälle überlebt hatte. Böcklin greift in seinem Werk immer wieder das Todesmotiv auf. Diese Häufung resultiert wohl auch aus diesen persönlichen Erfahrungen und nicht allein aus der damals herrschenden gründerzeitlichen Vorliebe für Endzeitstimmungen. „Das Bild muss so still werden, dass man erschrickt, wenn an die Tür gepocht wird“, soll Böcklin über sein Bild „Die Toteninsel“ gesagt haben.

\section{Ein Jahr „Psychoonkologie in Serie"}

Seit Böcklins Zeiten wissen wir deutlich mehr über Krebserkrankungen sowie den Bedarf und Nutzen einer psychoonkologischen Krankenbegleitung. Aber wir befinden uns noch nicht dort, wo wir mit der Betreuung (nicht nur) tumorkranker $\mathrm{Pa}$ tienten sein wollen. Denn es gibt sie noch die schmerzvollen Momente, wenn sich Krebskranke und ihre Angehörigen fragen, ob die im Nationalen Krebsplan und den in der S3-Leitlinie „Psychoonkologische Diagnostik, Beratung und Behandlung er- wachsener Krebspatienten“ festgelegten Behandlungsziele das Papier wert sind, auf dem sie geschrieben stehen.

Die Redaktion hat diese Ambivalenz zwischen Aufbruch und Verzweiflung zum Anlass genommen, sich in diesem Jahr in einer Serie mit wichtigen Fragen der Psychoonkologie zu beschäftigen. In dieser Ausgabe beginnen wir auf Seite 26 mit einer Bestandsaufnahme der „Versorgungslandschaft in der Psychoonkologie“.

\section{Von Versorgungsforschung bis Männergesundheit}

In den kommenden Ausgaben der „Onkologischen Welt" werden wir Konzepte zum Begriff des „existenziellen Leidens“ im Rahmen der multidimensionalen gesundheitsbezogenen Lebensqualität, Trends in der ambulanten psychoonkologischen Versorgung, Ansätze für einzeltherapeutische Kurzzeitinterventionen bis hin zur Hypnose und Hypnotherapie vorstellen. $\mathrm{Zu}$ wenig berücksichtigt sind auch psychoonkologische Spezifika adoleszenter und junger Erwachsener mit Krebs.

Und schließlich wagen wir uns an ein besonders kritisches Thema - dem männlichen Tumorpatienten. Männer leiden oft unsichtbar, tragen aber den Kampf mit ihrer Erkrankung in ihrem Inneren dafür umso nachhaltiger aus.

Ein Jahr ist eigentlich viel zu kurz, um sich mit der Psychoonkologie zu beschäftigen. Aber man muss schließlich einmal damit anfangen.

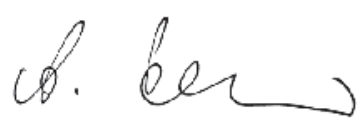

Dr. Alexander Kretzschmar, München

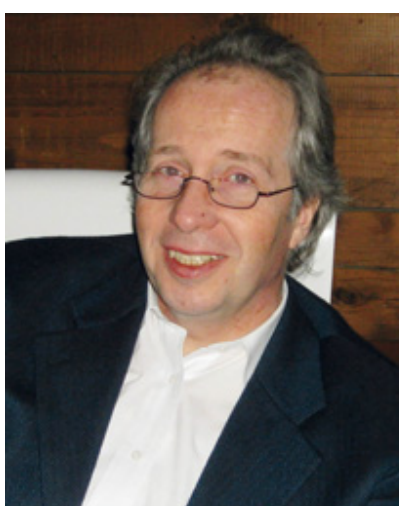

Dr. Alexander Kretzschmar 